\title{
Study on Evaluation Indicators System of Crowd Management for Transfer Stations Based on Pedestrian Simulation
}

\author{
Guanghou Zhang* \\ Transportation Research Center, Beijing University of Technology, Beijing 100124, China \\ Yanyan Chen \\ Transportation Research Center, Beijing University of Technology, Beijing 100124, China \\ E-mail: cdyan@bjut.edu.cn \\ www.bjut.edu.cn \\ Pingpu Li \\ Transportation Research Center, Beijing University of Technology, Beijing 100124, China \\ E-mail: liping@emails.bjut.edu.cn \\ www.bjut.edu.cn \\ Simon Fibbe \\ Westplantsoen 6B, 2613 GL Delft, the Netherlands \\ E-mail:S.T.Fibbe@student.tudelft.nl \\ www.transport.citg.tudelft.nl \\ Received 11 July 2011 \\ Accepted 25 November 2011
}

\begin{abstract}
Improving safety and convenience of transfer is one of the most vital tasks in subway system planning, design and operation management. Because of complicated space layout and crowded pedestrian, crowd control is a big challenge for management of transfer stations. Thus, a quantitative evaluation should be done before improvement measures are carried out. Literature review showed that present evaluation indicators about crowd management in subway system were all based on fixed value or experience. Dynamic effect caused by pedestrian congestion and various facility combination cannot be represented based on these indicators. Thus, in this paper, based on the pedestrian simulation tool, dynamic evaluation indicators system of crowd management was established from the point of safety, cost-effectiveness and comfort. In order to aid decision makers to identify the most appropriate scenario to improve the effectiveness of crowd management, Matter-Element Analysis (MEA) was used to rate different scenarios. A pedestrian simulation model of a designing intermodal transfer station was built and four different scenarios were tested to demonstrate how to use this indicators system. Simulation results were evaluated based on the dynamic indicators system and MEA. The application results show that the dynamic evaluation indicators system is operational and can reflect level of the crowd management in transfer station comprehensively and precisely.
\end{abstract}

Keywords: crowd management, dynamic evaluation indicators, pedestrian simulation, Matter-Element Analysis

\section{Background}

With the continuous improvement of the subway network system in Beijing, passenger volume increased dramatically. And with the subway system covering more and more areas, most of the passengers need to transfer between different lines. Based on the statistic data, the transfer ratio is 1.2-1.5. Many passengers need

\footnotetext{
* Transportation Research Center, Beijing University of Technology, 100 Pingleyuan, Chaoyang District, Beijing, 100124, China

Emails:guanghouzhang@emails.bjut.edu.cn
} 
to transfer between lines. As convergences node of multi-lines, transfer stations need to assume more passengers than other stations. During peak time, there will be a mass arrival in a very short time and the transfer facilities face great press. Consequently, transfer in subway station is an uncomfortable and timeconsuming issue for passenger. And, for the management, it is a great challenge to keep the transfer stations safe. But, crowd management in transfer station is a systematic work, included facility plan, facility design, and pedestrian traffic flow organization. Usually, it is a combination of kinds of measures. So, a quantitative evaluation should be done before the countermeasure scenarios of crowd management are carried out.

In china, many researchers have pay attention to the operation evaluation of subway or multimodal transfer stations. From the view of planning coordination, passenger satisfaction and operation management effectiveness, Liu xiaoming [1] proposed operation evaluation indicators system for urban public transfer terminals. Though this system included several qualitative indicators, it was one of earliest research results in such field in china. Following the basic frame proposed by Liu xiaoming, many researchers [2-7] improved and expanded the evaluation indicators system for transfer stations or hubs gradually.

But, the previous research results of evaluation system for transfer stations still have two mainly imperfect points. Firstly, there are still some qualitative indicators in exiting evaluation system. Setting value of these indicators need to be finished by experts. Thus, qualitative indicators are limited to the experience of the experts or decision makers and the values might be not objective and convictive. Especially for the planning or unfinished transfer stations, future operation status can only be imaged by experts. It is quite difficult to figure out results of different scenarios because of complicated facility layout and passenger behaviors. Secondly, calculations of the exiting quantitative indicators are all based on static threshold values in related design standard, such as, the speed of passenger and the capacity of facilities. Actually, different characteristics and behaviors of passengers will affect the capacity of all kinds of facilities. And, the demand of passengers is also assumed static and smooth. Generally, in procedure of facility design, the minimum interval used to count passenger volume is fifteen minutes. Actually, the headway for trains in subway system is much shorter than fifteen minutes. Thus, the wave of passenger flow, congestion and combination of kinds of facilities can dramatically affect the traffic behavior of passenger. Consequently, static evaluation can not reflect the real status of transfer stations.

Pedestrian simulation tools have been applied widely in crowd management. Especially in subway system, simulation tool can be used to evaluate the pros and cons of different scenarios such as facility design, passenger traffic organization and operation management [8-10]. With reasonable calibration of parameters and validation of output, pedestrian simulation tools can not only intuitively show the operational status of pedestrian traffic, but also record the accurate data about speed, acceleration, deceleration and track of all pedestrian. So, dynamic and quantitative evaluation can be done with pedestrian simulation tools. But, recently, qualitative description or basic comparison of simulation results is still the main application method of pedestrian simulation tools. The simulation evaluation method is not versatile because of lacking of standard dynamic evaluation indicators system and procedure.

Therefore, the goal of this paper was to propose a dynamic evaluation indicators system of crowd management in transfer stations based on Legion pedestrian simulation tool. The dynamic evaluation system selected indicators from the view of security, cost-effectiveness and comfort to evaluate different scenarios about crowd management in transfer stations.

\section{Evaluation Procedure Based on Pedestrian Simulation}

\subsection{Introduction of Legion Pedestrian Simulation Tool}

The multi-agent pedestrian model at the heart of the Legion simulation tool has been developed at the Maia Institute since 2000. Each pedestrian is modeled as a two-dimensional "entity" with a circular body, which moves in $2 \mathrm{D}$ continuous space, in $0.6 \mathrm{~s}$ time steps. The model can handle multiple floors, with special objects to enable the modeling of circulation elements such as stairs, escalators, ramps, moving walkways and lifts (elevators) [11].

Each entity moves towards its current target by selecting a step which seeks to minimize a perceived objective 
cost function. This cost is a weighted sum of the following three components:

- Inconvenience: Work to move, in excess of the amount which is necessary to reach one's destination;

- Frustration: Energetic cost equivalent of violating the speed preference time expenditure;

- Discomfort: Energetic cost equivalent of violating the preference clearance from other pedestrian and obstacles (Sum of functions of distances to predicted positions of perceived closest other pedestrian and other functions of distances to local obstacles).

Legion can record detailed track of each individual pedestrian and can accurately calculate the individual traffic behavior parameters and macroscopic traffic flow characteristics of pedestrian. The status of pedestrian traffic system can be reflected by maps, charts, tables. Detailed output parameters can be found in Table 1 .

Legion can be used to a lot of fields relevant with pedestrian. Such as pre-feasibility assessment, new station designs and station refurbishment projects. So, in this paper, evaluation indicators system was established based on the Legion studio 2006.
Table.1 Output parameters of Legion

\begin{tabular}{cc}
\hline Output format & Output parameters \\
\hline & Ingress and Egress \\
& Occupancy \\
Map & Flow \\
Chart & Speed \\
Table & Entity and Space density \\
& Journey time \\
& Social cost \\
& Inconvenience, Frustration, \\
& Discomfort and Dissatisfaction \\
\hline
\end{tabular}

\subsection{Evaluation procedure}

Traffic simulations model the complex traffic phenomenon and reproduce the actual traffic conditions based on predicting. Analysis works such as evaluation and optimization can be done based on simulation results. Fig. 1 shows the framework of evaluation procedure for crowd management in transfer stations based on pedestrian simulation. Analysis of simulation model can intuitively reflect the possible problems of the pedestrian facilities or pedestrian traffic organization in subway station. Then quantitative evaluation can be done with dynamic evaluation indicators system. At last, certain algorithms could be used to figure out the best scenario.

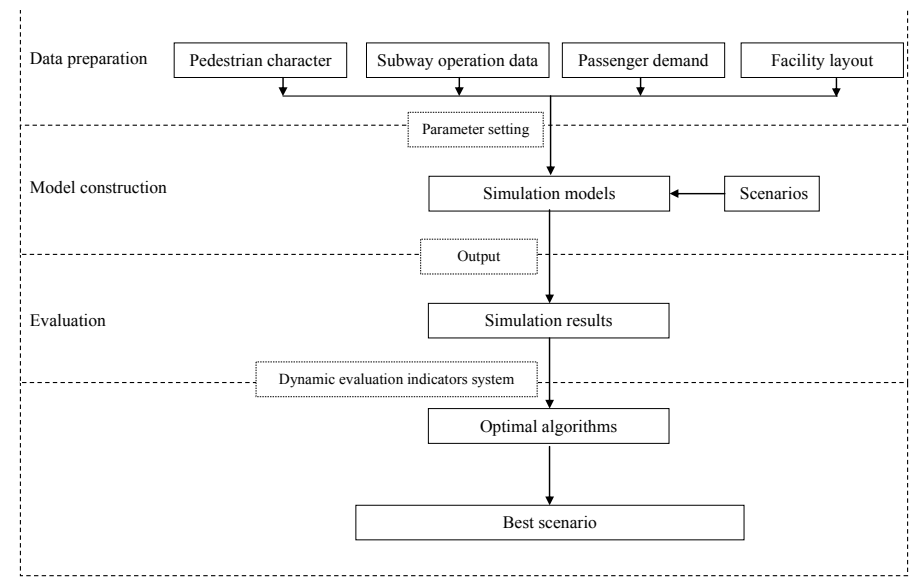

Fig. 1. Framework of evaluation procedure for crowd management in transfer stations based on pedestrian simulation

\section{Dynamic Evaluation Indicators System}

Making transfer more safe and efficient is the basic objective of crowd management in transfer stations. So, the main aim of dynamic evaluation of crowd management in transfer station is to evaluate the pros and cons of different scenarios in view of operation safety, transfer time and comfort. Therefore, this paper built dynamic evaluation indicators system based on the output results of Legion simulation software from the view of security, cost-effectiveness and comfort. The frame of this dynamic evaluation indicators system can be found in Fig.2. 


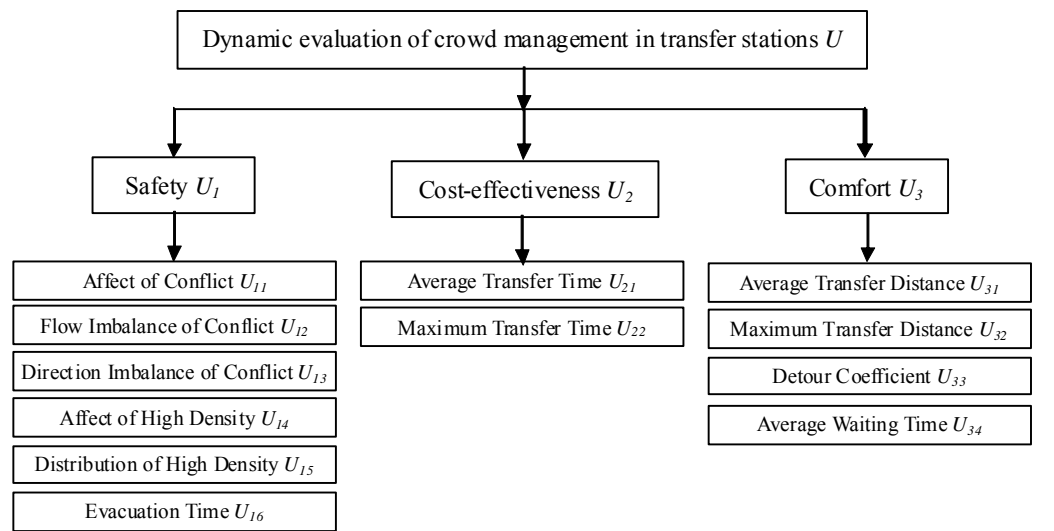

Fig. 2. Dynamic evaluation indicators system of crowd management for transfer stations

\subsection{Indicators of Safety}

\subsubsection{Affect of conflict $u_{11}$}

In transfer stations, crossing area with passengers from two or more directions is defined as conflict point. Affect of conflict points is represented by proportion of passengers who cross the conflict points. It is assumed as in Eq. (1):

$$
u_{11}=\sum_{i=1}^{n} q_{i} / q_{t}
$$

Where $q_{i}$ is passenger flow of conflict point $i, q_{t}$ is the total flow in the transfer station during simulation time, $n$ is the number of conflict points.

\subsubsection{Flow imbalance of conflict $u_{12}$}

This indicator reflects the flow distribution among different conflict points. Equilibrium distribution of passenger flow is helpful for safety of transfer stations. The more evenly the flow is distributed among different conflict points, the safer the transfer stations will be. Gini-Concentration index was used to calculate flow imbalance of conflict. Based on the definition, 0 means the distribution is most even; 1 means the distribution is dramatic concentration [12], as shown in Eq. (2):

$$
u_{12}=\sum_{i=1}^{n-1}\left|f_{i}-p_{i}\right| / \sum_{i=1}^{n-1} f_{i}
$$

Where $n$ is the number of conflict points, $f_{i}$ is accumulative distribution assumed that each conflict point has the same number of passengers, $f_{i}=i / n ; p_{i}$ is the actually accumulative distribution, $p_{i}=\sum_{j=1}^{i} q_{j} / \sum_{i=1}^{n} q_{i}, q_{i}$ is the real passenger flow of conflict point $j$.

\subsubsection{Direction Imbalance of Conflict $u_{13}$}

For a conflict point, evenly flow distributions of different directions means higher lose of capacity. This indicator reflects the flow distribution among different directions in one conflict point, as shown in Eq. (3):

$$
u_{13}=\sum_{i=1}^{n} w_{i} \cdot d_{i}
$$

Where $n$ is the number of conflict points, $w_{i}$ is weight of conflict point $i, d_{i}$ is the direction imbalance of conflict point $i . d_{i}$ can be also calculated based on GiniConcentration index, as follows:

$$
d_{i}=\sum_{j=1}^{d n_{n}^{-1}}\left|f_{i j}-p_{i j}\right| / \sum_{j=1}^{d n_{i}^{-1}} f_{i j}
$$

Where $d n_{i}$ is number of directions of passenger flow in conflict point $i, f_{i j}$ is accumulative distribution assumed that the distribution among all directions is even in conflict point $i, f_{i j}=j / d n_{i} ; p_{i j}$ is actually accumulative distribution among all directions in conflict point $i, p_{i j}=\sum_{m=1}^{j} q d_{i m} / q_{i}, q d_{i m}$ is passenger flow of direction $m$ in conflict $i, q_{i}$ is the real passenger flow of conflict point.

Considering number of directions and flow of different conflict points, $w_{i}$ can be calculated based on entropy weight coefficient method. Based on information theory [13], the entropy of conflict $i$ is:

$$
H_{i}=-\frac{1}{\ln d n_{i}} \sum_{j=1}^{d n_{i}} h_{i j} \ln h_{i j}
$$

Where, $h_{i j}=q d_{i j} / \sum_{k=1}^{d n_{i}} q d_{i k}$, the weight of conflict $i$ is: 


$$
W_{i}=1-H_{i} / n-\sum_{i=1}^{n} H_{i}
$$

\subsubsection{Affect of high density $u_{14}$}

Concentration of high-density crowd is a serious risk of safe for transfer stations. This indicator reflects the affect of high density. It is assumed that the value of high density is under D Level of service [14]. Considering duration and flow of affected passenger, the affect of high density is:

$$
u_{14}=\sum_{i=1}^{e} \sum_{j=1}^{m_{i}} l_{i j} \cdot v_{i j} / T_{t} \cdot q_{t}
$$

Where $e$ is the number of high density areas, $m_{i}$ is the number of high density duration in high density area $i, l_{i j}$ is length of high density duration $j$ in high density area $i$, $v_{i j}$ is passenger flow of high density duration $j$ in density area $i, T_{t}$ is total length of simulation time, $q_{t}$ is the total flow of the transfers station during simulation time.

\subsubsection{Distribution of high density $u_{15}$}

This indicator reflects distribution of passenger who suffered high density [14]. And the value of this indicator can be calculated by Legion directly.

\subsubsection{Evacuation time $u_{16}$}

Evacuation time is direct evaluation of evacuation ability in emergency. Because Legion can record the track of each pedestrian, the value of this indicator can be calculated by Legion software directly too.

\subsection{Indicators of Cost-effectiveness}

\subsubsection{Average transfer time $u_{21}$}

From the view of passenger, transfer time can intuitively reflect cost-effectiveness of transfer, as shown in Eq. (8):

$$
u_{21}=\sum_{i=1}^{q_{i}} t_{i} / q_{t}
$$

Where $t_{i}$ is transfer time of passenger $i, q_{t}$ is the total flow in the transfer station during simulation time.

\subsubsection{Maximum average transfer time $u_{22}$}

Transfer stations service kinds of transportation mode. It is necessary to make sure that transfer time of any OD pair cannot exceed a threshold value. This indicator reflects the maximum average transfer time of different transportation mode or OD pair, as shown in Eq. (9):

$$
u_{22}=\max _{1 \leq i \leq r} \max _{1 \leq j \leq s}\left\{\sum_{k=1}^{O_{i j}} t_{i j k} / O_{i j}\right\}
$$

Where $r$ is number of origin in simulation model, $s$ is number of destination in simulation model, $o_{i j}$ is passenger flow from origin $i$ to destination $j, t_{i j k}$ walking time of passenger $k$ from origin $i$ to destination $j$.

\subsection{Indicators of Comfort}

\subsubsection{Average transfer distance $u_{31}$}

Shorter transfer distance means passengers can easily arrive at their destinations. In other words, passenger will feel comfort if they could easily transfer. This indicator reflects the average transfer distance of all passengers, as shown in Eq. (10):

$$
u_{31}=\sum_{i=1}^{q_{i}} I_{i} / q_{t}
$$

Where $l_{i}$ is transfer distance of passenger $i, q_{t}$ is the total flow of the transfers station during simulation time.

\subsubsection{Maximum average transfer distance $u_{32}$}

As mentioned above, transfer stations cover kinds of transportation mode. It is also necessary to make sure that transfer distance of any OD pair cannot exceed a threshold value. This indicator reflects the maximum average transfer distance of different transportation mode or OD pair, as shown in Eq. (11):

$$
u_{32}=\max _{1 \leq i \leq r} \max _{1 \leq j \leq s}\left\{\sum_{k=1}^{O_{i j}} l_{i j k} / O_{i j}\right\}
$$

Where $r$ is number of origins in simulation model, $s$ is number of destinations in simulation model, $o_{i j}$ is passenger flow from origin $i$ and destination $j, l_{i j k}$ is the transfer distance of passenger $k$ from origin $i$ to destination $j$.

\subsubsection{Detour coefficient $u_{33}$}

This indicator reflects the transfer convenience of all passengers, as shown in Eq. (12):

$$
\boldsymbol{U}_{33}=\sum_{i=1}^{r} \sum_{j=1}^{s} W_{i j} \sum_{k=1}^{O_{i}} l_{i j k} / \boldsymbol{O}_{i j} \cdot S_{i j}
$$

Where $r$ is number of origin in simulation model, $s$ is number of destination in simulation model, $o_{i j}$ is passenger flow from origin $i$ and destination $j, l_{i j k}$ is the transfer distance of passenger $k$ from origin $i$ to destination $j, s_{i j}$ is the shortest transfer distance from origin $i$ to destination $j$.

$w_{i j}$ is weight of original $i$ and destination $j$, considering shortest distance and passenger flow of each OD pair, $w_{i j}$ can also be calculated based on entropy weight coefficient method which can be found from Eq. (5) and Eq. (6). 


\subsubsection{Average waiting time $u_{34}$}

Waiting time reflects the level of service of pedestrian service facilities such as ticket window, ticket machine, and security check. This indicator reflects the average waiting time of all passengers in transfer station, as shown in Eq. (13):

$$
u_{34}=\sum_{i=1}^{n} \sum_{j=1}^{p_{i}} w_{i j} / \sum_{i=1}^{n} p_{i}
$$

Where $n$ is the number of service facilities in transfer station, $p_{i}$ is the passenger flow of service facility $i, w_{i j}$ is waiting time of passenger $j$ in service facility $i$.

\section{Application}

In order to test the practicability of the dynamic evaluation system, this paper built a pedestrian simulation model of an intermodal transfer station and modeled four different scenarios about crowd management.

\subsection{Pedestrian Simulation Model}

The selected transfer station is SONGJIAZHUANG (SJZ) station in Beijing subway network. SJZ is a multimodels transfer hub. Passenger come from subway, bus, long-distance bus, taxi and private car will transfer here. It is expected that, by 2016, 792,000 passengers will be transfer here a day. And, in the peak hours, about 113,200 passengers will transfer. It is undoubted that SJZ transfer hub will be one of the busiest hubs in Asia when it is finished. So, it is very necessary to evaluate the scenarios of facility design, pedestrian traffic organization and headway schedule before construction. Data preparation is the basic work for pedestrian simulation modeling. Generally, for each subway station, surrounding land use and facilities layout are quite different. So, pedestrian traffic characteristics and pedestrian flow organization plans are also different for each station. Therefore, in order to build a simulation model more close to the real situation, exact data preparation work need to be done from the view of pedestrian traffic characteristics, facility layout, operational organization and other aspects, as shown in Table 2. Detail information about the data used in the simulation model can be found from the previous research work [8].

Based on the simulation results, the dynamic evaluation indicators were calculated, as shown in Table 3.

Table 2 Data preparation for pedestrian simulation modeling

\begin{tabular}{cc}
\hline Data & Contents \\
\hline Pedestrian characteristics & Speed distribution, route choice model, reaction of congestion and shopping time \\
Capacity of facilities & Auto-fare gate, security check, ticket window, ticket machine and checkout counters \\
Demand & Pedestrian traffic composition, arrival pattern, and OD distribution \\
Operation data of subway & Schedule, alight and boarding time \\
Facilities layout & Layout of pedestrian facilities \\
Pedestrian organization & Temp or Permanent fence, operation of Auto-fare gate, stairs, elevators or escalators \\
\hline
\end{tabular}

\subsection{Calculation for best scenario}

Many algorithms can be used to sort the best scenario, such as cosine function, linear assignment, matterelement analysis [15] and fuzzy logic[16-17]. This paper used matter-element analysis to calculate the value of decision-making of four scenarios based on the dynamic evaluation indicators.

The Matter-Element Analysis (MEA) is proposed to handle problems with contradictions and incompatibility using a set of matter element based correlation transformations [18]. The main theories of this analysis are the definition of matter elements, extension mathematics, and the matter element transformation theory. A matter element is a representation of the characteristics of the object under study, which can be defined using an ordered triad such as $\mathrm{ME}=(\mathrm{N}, \mathrm{C}, \mathrm{V})$. Where $\mathrm{N}$ denotes the name of the matter, $\mathrm{C}$ is its characteristic (or representative parameter), and $\mathrm{V}$ is called the "Field" session to store the measure of the characteristic, which can be a number, an interval, or a verbal description, etc. For matter elements with multiple parameters, $\mathrm{C}$ and $\mathrm{V}$ are represented as vectors [19], as shown in Eq. (14):

$$
M E=(N, C, V)=\left[\begin{array}{ccc}
N & C_{1} & V_{1} \\
& C_{2} & V_{2} \\
& \cdots & \cdots \\
& C_{n} & V_{n}
\end{array}\right]
$$

Thus the matter elements of the four scenarios can be represented as Eq. (15): 


$$
M E_{\text {original }}=\left[\begin{array}{ccccc} 
& S_{1} & S_{2} & S_{3} & S_{4} \\
U_{11} & 1.75 & 1.82 & 1.89 & 1.67 \\
U_{12} & 0.462 & 0.523 & 0.477 & 0.49 \\
U_{13} & 0.325 & 0.467 & 0.254 & 0.355 \\
U_{14} & 0.845 & 0.883 & 0.965 & 0.674 \\
U_{15} & 0.24 & 0.33 & 0.27 & 0.29 \\
U_{16} & 348 & 330 & 336 & 354 \\
U_{21} & 426 & 390 & 324 & 450 \\
U_{22} & 306 & 288 & 198 & 234 \\
U_{31} & 102 & 116 & 83 & 115 \\
U_{32} & 176 & 203 & 168 & 196 \\
U_{33} & 1.34 & 1.58 & 1.43 & 1.18 \\
U_{34} & 186 & 222 & 198 & 246
\end{array}\right]
$$

Because each element may be expressed in different units, a normalization process is generally applied, as Eq. (16):

$$
M E_{\text {normalized }}=\left[\begin{array}{ccccc} 
& S_{1} & S_{2} & S_{3} & S_{4} \\
u_{11} & 0.636 & 0.318 & 0.000 & 1.000 \\
u_{12} & 1.000 & 0.000 & 0.754 & 0.541 \\
u_{13} & 0.333 & 1.000 & 0.000 & 0.474 \\
u_{14} & 0.412 & 0.282 & 0.000 & 1.000 \\
u_{15} & 1.000 & 0.000 & 0.667 & 0.444 \\
u_{16} & 0.250 & 1.000 & 0.750 & 0.000 \\
u_{21} & 0.190 & 0.476 & 1.000 & 0.000 \\
u_{22} & 0.000 & 0.167 & 1.000 & 0.667 \\
u_{31} & 0.424 & 0.000 & 1.000 & 0.030 \\
u_{32} & 0.771 & 0.000 & 1.000 & 0.200 \\
u_{33} & 0.600 & 0.000 & 0.375 & 1.000 \\
u_{34} & 1.000 & 0.400 & 0.800 & 0.000
\end{array}\right]
$$

Thus, the standard matter element is defined as Eq. (17):

$$
M E_{\text {standard }}=\left[\begin{array}{ccc} 
& S_{-} & S_{+} \\
U_{11} & 0 & 1 \\
U_{12} & 0 & 1 \\
U_{13} & 0 & 1 \\
U_{14} & 0 & 1 \\
U_{15} & 0 & 1 \\
U_{16} & 0 & 1 \\
\boldsymbol{U}_{21} & 0 & 1 \\
\boldsymbol{U}_{22} & 0 & 1 \\
\boldsymbol{U}_{31} & 0 & 1 \\
\boldsymbol{U}_{32} & 0 & 1 \\
\boldsymbol{U}_{33} & 0 & 1 \\
\boldsymbol{U}_{34} & 0 & 1
\end{array}\right]
$$

So the decision value of scenario $i$ can be calculated based on the following Eq. (18):

$$
S_{i}=\sum_{j=1}^{P I}\left[w_{j}\left(S_{+}-S_{i j}\right)\right]^{2}-\sum_{j=1}^{P I}\left[w_{j}\left(S_{i j}-S_{-}\right)\right]^{2}
$$

Where $P I$ is the number of indicators in transfer station, $S_{i j}$ is the normalized value of indicator $j$ in scenario $i, w_{j}$ is weight of indicator $j, w_{j}$ can also be calculated based on entropy weight coefficient method which can be found from Eq. (5) and Eq. (6).

The result was: $S_{1}=-0.99581, S_{2}=-0.12445, S_{3}=-0.65961$, $S_{4}=0.01604$. Because $S_{4}>S_{2}>S_{3}>S_{1}$, so the best scenario is Scenario 1.

Table.3 Evaluation indicator values of different scenarios

\begin{tabular}{ccccc}
\hline Evaluation indicators & Scenario $1\left(S_{1}\right)$ & Scenario $2\left(S_{2}\right)$ & Scenario 3( $\left.S_{3}\right)$ & Scenario $4\left(S_{4}\right)$ \\
\hline Affect of conflict $u_{11}$ & 1.75 & 1.82 & 1.89 & 1.67 \\
Flow imbalance of conflict $u_{12}$ & 0.462 & 0.523 & 0.477 & 0.49 \\
Direction Imbalance of Conflict $u_{13}$ & 0.325 & 0.467 & 0.254 & 0.355 \\
Affect of high density $u_{14}$ & 0.845 & 0.883 & 0.965 & 0.674 \\
Distribution of high density $u_{15}$ & 0.24 & 0.33 & 0.27 & 0.29 \\
Evacuation time $u_{16}$ & 348 & 330 & 336 & 354 \\
Average transfer time $u_{21}$ & 426 & 390 & 324 & 450 \\
Maximum average transfer time $u_{22}$ & 306 & 288 & 198 & 234 \\
Average transfer distance $u_{31}$ & 102 & 116 & 83 & 115 \\
Maximum average transfer distance $u_{32}$ & 176 & 203 & 168 & 196 \\
Detour coefficient $u_{33}$ & 1.34 & 1.58 & 1.43 & 1.18 \\
Average waiting time $u_{34}$ & 186 & 222 & 198 & 246 \\
\hline
\end{tabular}

\section{Conclusion}

Developing transit system can effectively solve the traffic congestion problem in big cities. Improving safety and convenience of transfer can attract more people to use public transport. It is useful to enhance level of service of urban public transport and keep the sustainable development of urban transport system in china. In this paper, a dynamic evaluation indicators 
system for crowd management in transfer stations was proposed based on pedestrian simulation tool. Practical application showed that the dynamic evaluation indicators system could reflect pedestrian traffic status in transfer station accurately and it could be used to quantitatively evaluate the pros and cons of pedestrian facilities planning and layout, traffic organization and operations management in transfer station. Actually, except transfer stations, it could be used to evaluate crowd management in other place, such as subway station, commercial building and venues for special events.

\section{Acknowledgements}

This paper was supported by The Technology Program of Beijing (No. D101100049410002) and Foundation for Key Laboratory of traffic Engineering of Beijing University of Technology (No. JTP-2011-07).

\section{References}

1. X. M. LIU, L. L. SHEN and X. L. YANG, Overall Evaluation Indìcator of Urban Passenger Transfer Hub, China Journal of Highway and Transport. 1(8)(1995)97102.

2. X. H. FANG, L. S. ZHOU and B. ZHOU, Evaluation Method of City Comprehensive Transportation Hub, Journal of Transportation Systems Engineering and Information Technology. 8(2)(2008)108-113.

3. T. ZONG, S. H. ZHANG and D. F. HUO, Evaluation Research on Urban Passenger Transfer Hub, Journal of Water Resources and Architectural Engineering. 5(3)(2007) 68-71.

4. W. LI, W. WANG and W. DENG, Grey Relationship Multi-Criteria Appraising Method Study of the Evaluation for Urban Passenger Transfer Hub, Journal of Transportation Engineering and Information. 4(12)(2004) 5-9.

5. L. S. SUN, J. RONG and F. T. REN, DEA Method for Evaluating the Transfer Efficiency of Urban Public Transportation Terminal, Journal of Beijing University of Technology. 33(12)(2007)1289-1294.

6. Q. G. Wu and L. Liu, Evaluation of Urban Mass Transit Transfer Hub based on Information Granules, Urban Mass Transit. 11(2008) 21-24.

7. C. Y. GUAN and Z. R. WANG, Application of DEA in Evaluation for Urban Passenger Transfer Hubs, Journal of Highway and Transportation Research and Development. 25(9)(2009)145-149.

8. G. H. ZHANG, Y. Y. CHEN and D. C. WU, Study on Pedestrian Traffic Characteristics of Transfer Hubs in Beijing, in Proceedings of 89th Annual Meeting of the Transportation Research Board (Compendium of Papers, CD-ROM), Washington D.C, 2010.
9. X. H. MIAO, Study on the Evaluation of Metro-hub Based on the Dynamic Simulation of Passenger, $P h D$ Thesis, Beijing: School of Civil Engineering, Beijing Jiaotong University, 2008.

10. M. W. HU and Q. X. SHI, Simulation and Evaluation of Passenger Flow Organization in Urban Rail Transit, Journal of Transportation Information and Safety. 27(3)(2009)39-42.

11. J. J. Berrou, J. Beecham and P. Quaglia, Calibration and validation of the Legion simulation model using empirical data, in Proceedings of Pedestrian and Evacuation Dynamics 2005 , Springer Berlin Heidelberg. 3(2007)167-181.

12. J. G. SHI, Research on Pedestrian Traffic Characteristics in Special Events, $P h D$ Thesis, Beijing: College of Architecture and Civil Engineering, Beijing University of Technology, 2007.

13. Q. L. YANG and Y. B. GUO, Application of the Fuzzy Matter-element Model Based on Coefficients of Entropy to Evaluation of Urban Eco-security, Mathematics in Practice and Theory. 40(19)(2010)62-67.

14. J. J. Fruin, Pedestrian Planning and Design. (Metropolitan Association of Urban Designers and Environmental Planners, Inc., New York, 1971)

15. Q. Z. HU and W. DENG, Optimization Models and Rating Methods of Urban Public Transport. (Beijing: Science Press , 2009)

16. V. López, M. Santos, J. Montero, Fuzzy Specification in Real Estate Market Decision, International Journal of Computational Intelligence Systems. 3 (1)(2010)8-20.

17. M. Ekmekçioğlu, A. C. Kutlu, C. Kahraman, A Fuzzy Multi-Criteria SWOT Analysis: An Application to Nuclear Power Plant Site Selection, International Journal of Computational Intelligence Systems. 4(4)(2011)583595.

18. W. Cai, Extension Engineering Methods.(Science Press, Beijing, China, 2003)

19. Q. LI, X. H. SHE, K. C. P. Wang, and K. D. Hall, An Exploration of Matter-Element Analysis for Pavement Preventive Maintenance Optimal Timing Determination and Treatment Selection, in Proceedings of 89th Annual Meeting of the Transportation Research Board (Compendium of Papers, CD-ROM), Washington D.C, 2010. 